\title{
Abnormal eye-head coordination in Parkinson's disease patients after administration of levodopa: a possible substrate of levodopa-induced dyskinesia
}

\author{
MICHAEL WEINRICH, RAJIV BHATIA \\ From the Department of Neurology, Palo Alto VA Medical Center, Stanford University Medical School, \\ Palo Alto, California, USA
}

SUMMARY Head oscillations in response to whole body oscillation of Parkinsonian patients before and after administration of levodopa were compared with those of normal subjects. Parkinsonian patients developed large increases in the amplitude of their head movements while fixating a stationary target after administration of levodopa. This increase was proportional to disease severity and was maximal in stage IV patients with levodopa induced dyskinesias. These findings indicate a fundamental yet reversible change in the strategy of eye-head coordination in Parkinsonian patients after administration of levodopa which may be due to release of normally inhibited visuo-vestibular reflexes.

Parkinsonian patients characteristically develop depressed righting reflexes ${ }^{1}$ and difficulty maintaining their balance. Complaints of falling frequently bring these patients to medical attention. These patients also develop impaired ability to suppress their vestibulo-ocular reflex (VOR) and may exhibit inappropriately large VOR gains when placed in situations requiring suppression. ${ }^{2}$ These findings suggest defective supranuclear control of vestibular reflexes in Parkinson's disease.

Many Parkinsonian patients develop dyskinesias after long term treatment with levodopa. ${ }^{3}$ DennyBrown $^{4}$ and Purdon-Martin ${ }^{5}$ have proposed that released vestibulospinal reflexes might contribute to dystonia. The vestibular system has potent connections to the neck musculature via the medial longitudinal fasciculus and the medial vestibulospinal tract. $^{6}$ To investigate the hypothesis that levodopainduced dyskinesias might be mediated by vestibular reflexes we have studied the dynamics of head oscillations induced by whole body oscillation of Parkinsonian subjects before and after medication with levodopa.

Address for reprint requests: Dr $M$ Weinrich, Department of Neurology, 127 Palo Alto VA Medical Center 3801 Miranda Blvd. Palo Alto, CA 94304, USA

Received 18 June 1985 and in final revised form 11 November 1985. Accepted 18 November 1985

\section{Methods}

Patients Sixteen patients were studied, and all were examined by a neurologist (MW) prior to entry into the study and the diagnosis of Parkinson's disease confirmed. Patients were graded on the Hoehn and $\mathrm{Yahr}^{7}$ scale of clinical severity. The table summarises the characteristics of the patient population for each group of disease severity (II-IV). On the day of investigation, patients were instructed to withhold their morning medication. They were then studied at $9 \mathrm{am}$ in the head and eye movement apparatus described below. (Two patients were unable to withhold completely medication and took doses of Sinemet at least $21 / 2$ hours prior to the study). After the pre-medication study was performed, the patients took their usual dose of Sinemet, but no other medication. The head and eye movement studies were repeated one hour after the patients took their medication.

Controls Ten normal subjects ages 58 to 84 years (mean 65) were also studied with the same protocol but no medication. These subjects were medical school senior faculty and staff in good health and normal volunteers. Normal volunteers all had normal neurological examinations and normal psychometric testing. Informed consent was obtained from all subjects prior to study.

Apparatus Patients were seated on a velocity servocontrolled rotating chair (Genisco 1185-3) and secured tightly to it with shoulder and waist straps. Head movement relative to the body in the horizontal plane (yaw) was measured with a magnetic search coil system ${ }^{8}$ which detected rotation of coils mounted on a lightweight $(50 \mathrm{gm})$ plastic helmet and on a strap secured around either the subject's chest or neck (see fig 1). Resolution of the system was better than 10 minutes of arc. Tilting the head $30^{\circ}$ and eccentric 
Table Clinical characteristics of patients

\begin{tabular}{lccc}
\hline Clinical stage & $I I$ & $I I I$ & $I V$ \\
\hline Age & $46-76(62)$ & $67-82(72)$ & $63-77(68)$ \\
Duration of disease $(\mathrm{yr})$ & $1-7(5)$ & $4-16(9)$ & $4-23(14)$ \\
Dose of levodopa $(\mathrm{mg})$ & $400-750(575)$ & $300-875(540)$ & $150-2250(1093)$ \\
$\mathrm{N}$ & 4 & 5 & 7 \\
\hline
\end{tabular}

placement of the head in the field in combination produced errors of less than $30 \%$. Correction factors were calculated for each subject each time the subject was seated in the chair by comparing the maximum voltage deflections produced by rotating the coil $180^{\circ}$ around the subject's head with that expected in the centre of the field. Linear motion of the coils within $10 \mathrm{~cm}$ of the centre of the field produced no discernible artifact when the angular position of the head was within $10^{\circ}$ of midposition. Eye position was measured with

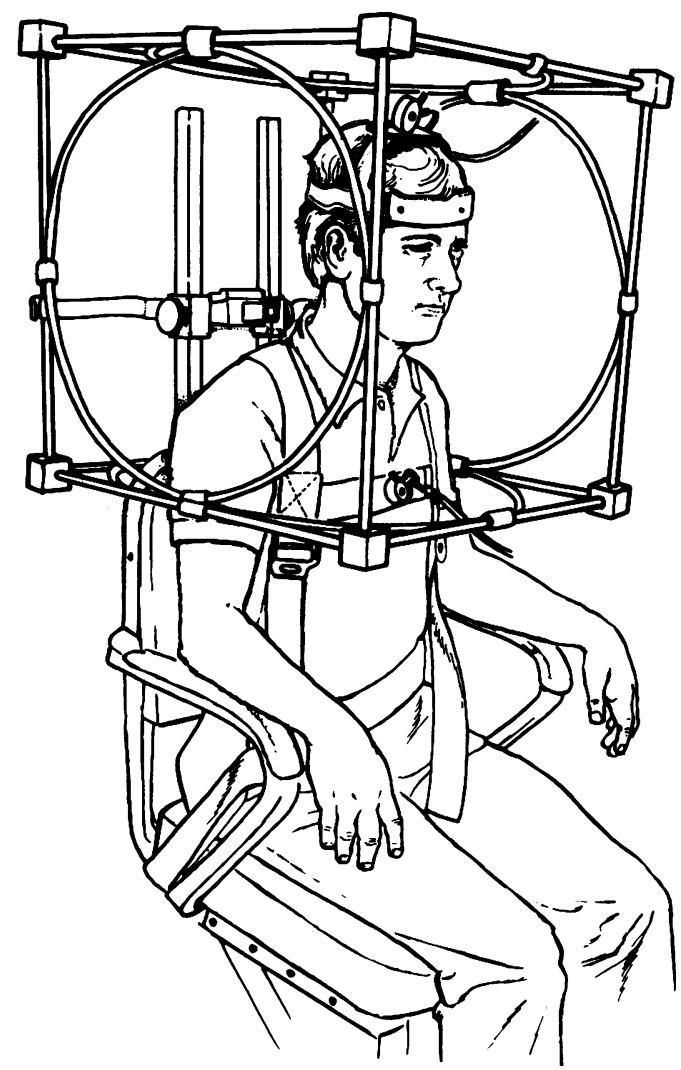

Fig 1 Apparatus for measuring head position relative to body position. The subjects sits on a velocity servo-controlled chair. Mounted on the chair are two pairs of Helmholtz coils which carry high frequency oscillating currents. The currents induced in the small coils mounted on the subject's head and neck (or chest as shown) are proportional to the angle between the axes of the large and small coils. The net angle between head and body is the subtracted signal of the two small coils. electro-oculography (bandpass 0.01 to $30 \mathrm{~Hz}$ ). Head position, eye position and chair velocity were low pass filtered $(10 \mathrm{~Hz})$ digitised with 12 bit resolution at $50 \mathrm{~Hz}$ and stored on floppy discs for offline analysis by a Nicolet $12 / 70$ computer.

Protocol All subjects were studied with their visual acuity corrected by their normal correction for distance. They were instructed to fixate either a $1 \mathrm{~cm}$ diameter spot on the wall $1.8 \mathrm{~m}$ from their nasion (target stationary in space) or the end of a rigid rod mounted on the chair so that its tip was 65 $\mathrm{cm}$ from their nasion (target moving with the subject). Subjects were instructed to "lock their eyes on the target". Some subjects asked for further instructions and were told to fixate the target in what seemed the most natural way to them. We deliberately avoided any suggestions to the patients on what strategy of eye-head coordination they should choose. The chair was oscillated at $30^{\circ} / \mathrm{s}$ at frequencies of 0.4 to $1.0 \mathrm{~Hz}$ in $0.2 \mathrm{~Hz}$ steps. Data were acquired for forty seconds at each frequency in the two conditions. The data from each trial were displayed on a CRT terminal immediately. Trials in which the subjects talked, became drowsy or distracted were rejected.

Analysis Fourier analysis of the data was performed off-line. At each frequency of chair oscillation, the component of head movement (relative to the body) at the frequency of chair oscillation was extracted. Gain and phase of head velocity with respect to the chair velocity were computed.

\section{Results}

Figure 2 illustrates the response of a normal subject to whole body oscillations. The head movements are composed predominantly of a sinusoidal oscillation related to the chair oscillation which is approximately in phase with the subject's eye movements but usually less than $10 \%$ of their amplitude. Thus, smooth pursuit eye movements are largely responsible for tracking the target and the eye and head position sum appropriately to compensate for chair movement. Prior to ingestion of levodopa, Parkinsonian subjects behaved qualitatively much as normal subjects in these tests. Figure 3 illustrates a representative section of a recording from one patient with stage IV disease prior to medication. Note the qualitative similarity of the responses of this patient with those of the normal subject shown in fig 2.

In contrast, the strategy employed by this patient shifts dramatically after administration of levodopa. As seen in fig 4, after levodopa this patient's head moves opposite to the chair rotation as before, but the amplitude of the head movement has increased 

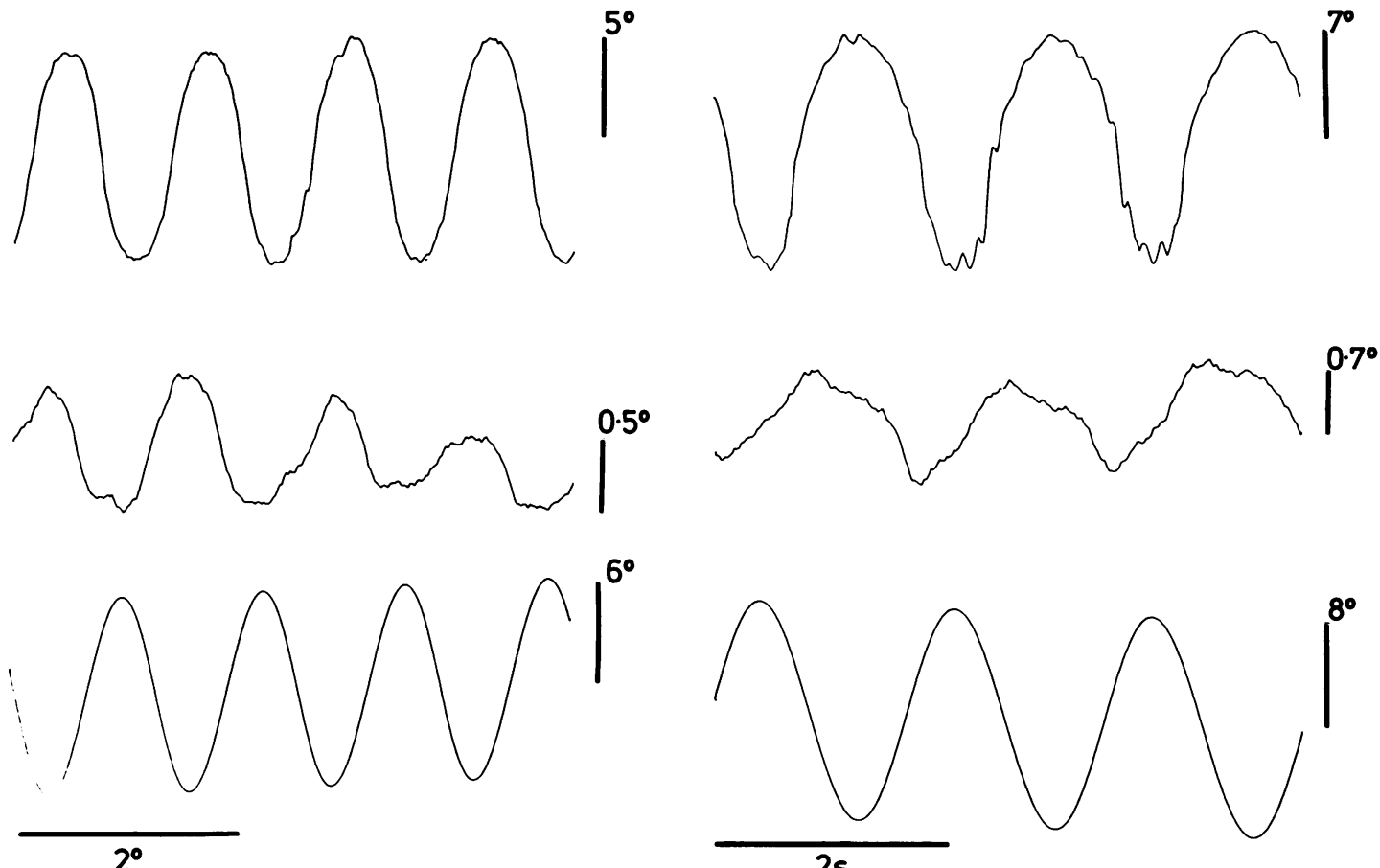

Fig 2 Head and eye movements of an 84-year-old normal volunteer. The subject fixated on a stationary target during chair oscillation. Top trace indicates eye position (EOG); middle trace indicates head position and the bottom trace indicates chair position. Note that smooth pursuit eye movements are largely responsible for tracking the target. Head movements are predominantly composed of an oscillation linearly related to the chair oscillation. These head oscillations are less than $10 \%$ of the amplitude of the eye movements and sum approximately with them to compensate for chair movement.

markedly, so that it actually exceeds the amplitude of chair movement. As shown in fig 4, the patient's eyes must move in the direction of the chair movement at a velocity which permits them to remain fixated on the target. Note that in this condition, the eye movements are opposite the direction of the vestibuloocular reflex.

Further insight can be obtained by examining this patient's head movements when he fixated a target moving with him. In this condition, optimal fixation is obtained if the subject's head and eyes remain motionless. Figure 5 shows this patient's head movements before and after medication while he fixated the target moving with him. Prior to medication (upper trace), head movements are quite small. After administration of levodopa (middle trace), the patient exhibited head movements over six times larger than the movements prior to medication. He was intermit-

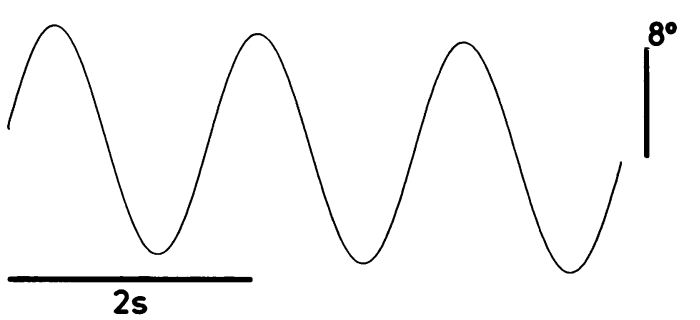

Fig 3 Head and eye movements in a patient with stage IV Parkinson's disease prior to medication while the subject fixated a stationary target. The top trace indicates eye position, the middle trace indicates head position and the bottom trace chair position. Note the similarity with the responses of the normal subject illustrated in fig 2 .

tently able partially to suppress these movements, but never decreased his head movements to less than twice the pre-medication level. In each case, eye movements (not shown) opposite to the head rotation, that is in the direction of chair rotation, compensated for the head movements.

Mean gains between head velocity and chair velocity over the four frequencies tested for all subjects while they fixated a stationary target are plotted in fig 6. Gain is expressed in $\mathrm{dB}\left(20^{*} \log\right.$ head velocity/chair velocity). Normal subjects in this study had relatively narrow range of gains; no normal subject had a mean gain higher than $-18 \mathrm{~dB}$. The mean gains of patients in all clinical groups before medication were not significantly different from controls although the variation was increased, especially in stage IV patients. There was little effect of levodopa on the head movements of stage II patients (mean change $1.9 \mathrm{~dB}$ ), a modest increase $(6.4 \mathrm{~dB})$ in the movements of stage III patients and a mean increase of $16.3 \mathrm{~dB}$ in the head movements of stage IV patients. This increase corresponds to an increase in amplitude of $553 \%$. The 


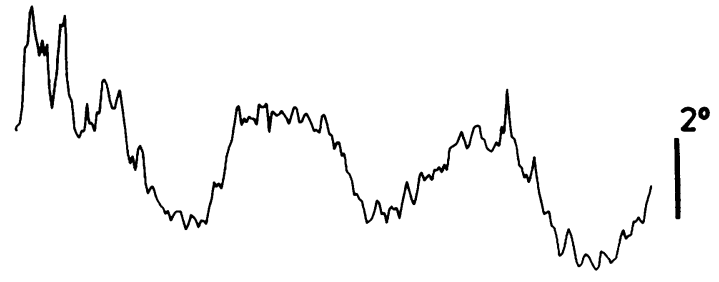

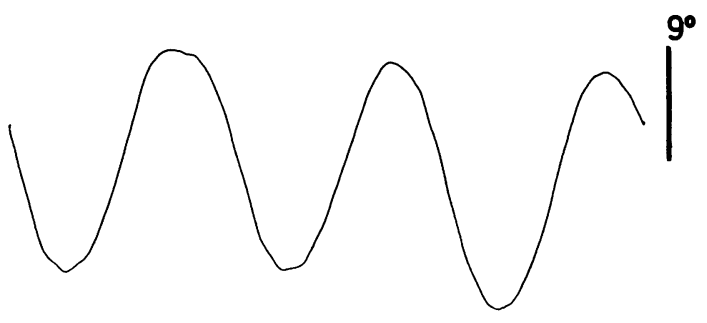

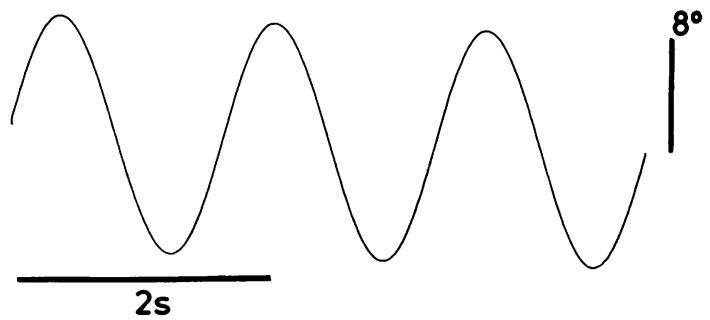

Fig 4 Head and eye movements in the same patient performing the identical task as in fig 3 after administration of levodopa. Traces represent the same signals. Note that the amplitude of the head oscillation has increased more than ten-fold and that eye movements have reversed in direction.

only stage IV patient who did not exhibit a marked increase in head movements could tolerate only $1 / 2$ of a 10/100 Sinemet tablet three times a day. Note that $4 / 7$ of the stage IV patients demonstrated head movements after medication which are more than two standard deviations above the normal range. Three of these patients had levodopa-induced dyskinesias, and the highest gains were seen in the two patients with the most prominent dyskinesias. Neither the mean gain nor the change in gain after medication predicted the occurrence of the milder dyskinesias seen in other patients; however, we were unable to control for the effects of other medications with longer half-lives, for example bromocriptine, which some patients were also taking.

\section{Discussion}

These data support the hypothesis that Parkinsonian patients treated with levodopa develop defective supranuclear control of head as well as eye movements.
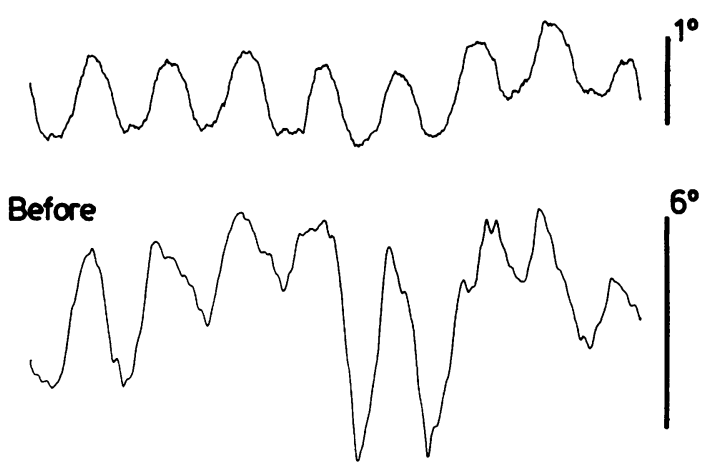

After

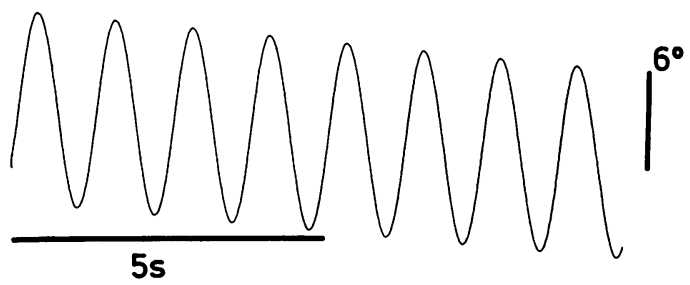

Fig 5 Head movements before and after levodopa in the same patient as in figs 3 and 4 while he fixated a target moving with him. Top trace indicates head movements prior to levodopa, middle trace indicates head movements after levodopa and the bottom trace indicates chair position. Note the large increase in amplitude of oscillations after levodopa and the intermittent success of the patient in suppressing them.

The head movements observed in these patients are $180^{\circ}$ out of phase with the vestibular stimulation and appear to be involuntary, and this impression is reinforced by the extreme examples observed when patients' head movements are actually serving to destabilise retinal images. Increased head movements in response to oscillation might be caused by the decreased smooth pursuit and saccadic function observed in Parkinsonian patients. ${ }^{9}$ However, in the examples shown, the eyes moved opposite to the direction of perceived target motion (opposite to normal pursuit and VOR direction). Involuntary head movements associated with saccadic eye movements between targets has been reported by Shimuzu et al ${ }^{10}$ in Parkinsonian patients; however, Kennard, et al ${ }^{11}$ found that Parkinsonian patients made little or no head movement when tracking predictable and unpredictable targets with "natural" eye and head movements. In neither study was the effect of levodopa investigated.

The strategy of eye-head coordination employed by Parkinsonian patients appears to shift reversibly with 


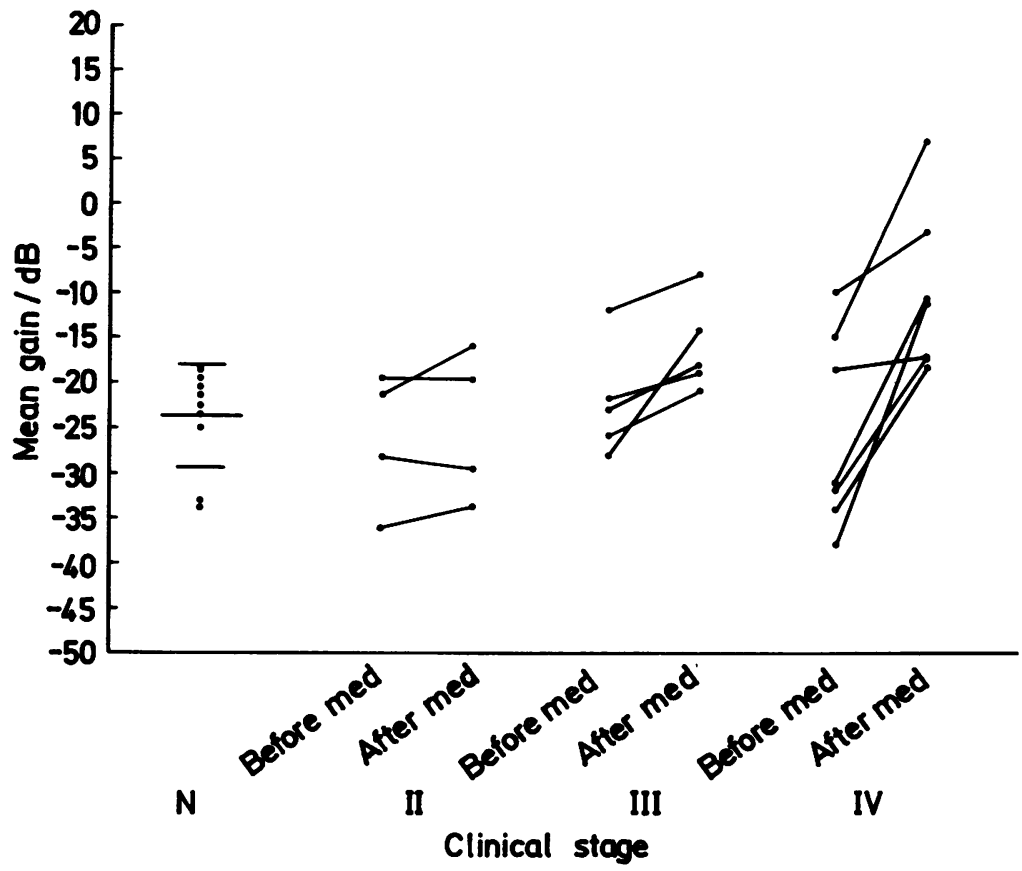

Fig 6 Mean gain between chair velocity and head velocity over the four frequencies tested for ten normal subjects in the same age range and Parkinsonian patients before and after levodopa. Mean and standard deviation for normals are indicated by horizontal lines. Note the progressive increase in gain after levodopa in patients with increasing severity of disease. The mean increase in head movement amplitude of stage IV patients is $553 \%$.

administration of levodopa. Decreasing the rigidity of the neck will increase the size of head movements in response to whole body oscillations, but tone would have to decrease considerably below normal to produce the increases observed. ${ }^{12}$ The large oscillations illustrated in fig 4 could have been produced if tone had decreased so much that the head and neck were in a state of mechanical resonance; however, in that case one would expect large spontaneous oscillations which were not observed. Further, the largest oscillations were observed in stage IV patients, some of whom had markedly increased neck stiffness prior to medication.

The reversibility of these increased head oscillations in Parkinsonian patients contrasts with the observations of Jenkyn et $a l,{ }^{13}$ who observed a "nucho-cephalic" reflex in demented patients. This reflex was elicited by "rapidly turning the shoulders of a standing subject to the right or left while his eyes are closed to avoid visual fixation." The experimental situation described here is quite different especially: in. regard to the relative strengths of vestibular and pro-. prioceptive input, and the visual input and instructions. Normal subjects can demonstrate markedly different VOR gains in darkness with different instructions. ${ }^{14}$ Thus, the increased head oscillations in Parkinsonian patients should not be equated with cognitive dysfunction, especially since these oscillations were dependent on administration of levodopa.

We can not distinguish between the possibilities that levodopa causes the release of visuo-vestibular reflexes in patients with advanced disease by stimulation of brainstem pathways or that the decrease in rigidity in these patients with levodopa unmasks a reflex that is already uninhibited. The relatively small effect of levodopa on stage II patients suggests that levodopa alone is not sufficient to cause the release of such reflexes. (One of us, M.W., also took levodopa with no effect on induced head oscillations.) Patients with advanced disease have increased difficulty with balance and defective righting reflexes and might be expected- to have defective supranuclear control of vestibulo-spinal reflexes but these patients also have 
generally had their disease longer and taken doses of levodopa higher than patients with milder disease. Thus, they are more likely to develop problems with dopamine receptor supersensitivity. ${ }^{3}$ Therefore, the interpretation of these results must be made with some caution as the effect of levodopa on the brainstems of these patients may be quite different from the effects on normal subjects or mildly affected patients. It is tempting to ascribe the increased oscillations in Parkinson's patients to an exaggerated vestibulospinal reflex; however, other systems might also play a role, particularly the recently observed modulation of neck muscle activation by horizontal eye position. ${ }^{1516}$

This work was supported by a Medical Merit Review grant from the Veteran's Administration. We thank Dr Ronald Angel for his helpful comments and suggestions.

\section{References}

${ }^{1}$ Fahn S, Duffy P. Parkinson's Disease. In: Goldensohn E, and Appel, SH, eds. Scientific Approaches to Clinical Neurology. Philadelphia: Lea and Febiger, 1977: 1119-58.

${ }^{2}$ White OB, Saint-Cyr R, Tomlinson RD, Sharpe JA. Ocular motor deficits in Parkinson's disease: I. The horizontal vestibulo-ocular reflex and its regulation. Brain 1983;106:555-70.

${ }^{3}$ Yahr MD. Limitations of long term use of antiparkinsonian drugs. Can J Neurol Sci 1984;11 (supp.): 191-4.

${ }^{4}$ Denny-Brown D. Clinical symptomatology of diseases of the basal ganglia. In: Vinken PJ and Bruyn GW, eds. Handbook of Clinical Neurology, vol. 6 Diseases of the Basal Ganglia. Amsterdam: North Holland 1968: 133-72.

${ }^{5}$ Purdon-Martin J. The Basal Ganglia and Posture. London: Pitman Medical 1967.

${ }^{6}$ Wilson VJ, Melville-Jones G. Mammalian vestibular physiology. New York: Plenum 1979:185-248.

${ }^{7}$ Hoehn MM, Yahr MD. Parkinsonism: Onset, progression, and mortality. Neurology (Minneap) 1967; 17:427-42.
${ }^{8}$ Remmel RS. An inexpensive eye movement monitor using the scleral search coil technique. IEEE Trans Biomed Eng 1984;BME-31:388-90.

${ }^{9}$ White OB, Saint-Cyr JA, Tomlinson RD, Sharpe JA. Ocular motor deficits in Parkinson's disease: II. Control of the saccadic and smooth pursuit systems. Brain 1983;106:571-88.

${ }^{10}$ Shimizu N, Naito M, Yoshida M. Eye-head coordination in patients with Parkinsonism and cerebellar ataxia. $J$ Neurol Neurosurg Psychiatry 1981;44:509-15.

${ }^{11}$ Kennard C, Zangmeister WH, Mellors S, Stark L, Hoyt WF. Eye-Head coordination in Parkinson's disease. In: Lennerstrand G, Zee D and Keller E, eds. Functional Basis of Ocular Motility Disorders, Proceedings of a Wenner Gren Center and Smith-Kettlewell Eye Research Foundation International Symposium Stockholm 1982:517-20.

12 Note: While the head-neck system is non-linear in response to different amplitude oscillations, at a given operating point the system can be approximately modeled as a second order system, i.e., a damped harmonic oscillator. Inspection of the equation for such a system reveals that the amplitude of oscillation is approximately inversely proportional to stiffness. The resonant frequency of the head-neck system in normal subjects is approximately $3 \mathrm{~Hz}$ - see Barnes, GR and Rance, GH. Transmission of angular acceleration to the head in the normal seated subject. Aerospace Medicine 1974; 45:411-6.

${ }^{13}$ Jenkyn LR, Walsh DB, Walsh BT, Culver CM, Reeves AG. The nuchocephalic reflex. J Neurol Neurosurg Psychiatry 1975;38:561-6.

${ }^{14}$ Collewijn H, Martins AJ, Steinman RM. Compensatory eye movements during active and passive head movements: fast adaptation to changes in visual magnification. J Physiol (Lond) 1983;340:259-86.

${ }^{15}$ Berthoz A, Vidal PP, Corvisier J. Brain stem neurons mediating horizontal eye position signals to dorsal neck muscles of the alert cat. In Roucoux $A$ and Crommelinck M eds. Physiological and pathological aspects of eye movements. The Hague M Junk 1982:385-98.

${ }^{16}$ Darlot C, Denise P, Droulez J. Modulation by horizontal eye position of the vestibulo-collic reflex induced by tilting in the frontal plane in the alert cat. Exp Brain Res 1985;58:510-9.

${ }^{17}$ Denny-Brown D, Yanagisawa N. The role of the basal ganglia in the initiation of movement. In: Yahr MD, ed. The Basal Ganglia. New York: Raven Press 1976: 115-49. 